УДК 517.95

\title{
On an Inverse Problem for Quasi-Linear Elliptic Equation
}

\author{
Anna Sh. Lyubanova* \\ Institute of Space and Information Technology \\ Siberian Federal University \\ Kirenskogo, 26, Krasnoyarsk, 660026
}

Russia

Received 12.11.2014, received in revised form 03.12.2014, accepted 20.12.2014

\begin{abstract}
The identification of an unknown constant coefficient in the main term of the partial differential equation $-k M \psi(u)+g(x) u=f(x)$ with the Dirichlet boundary condition is investigated. Here $\psi(u)$ is a nonlinear increasing function of $u, M$ is a linear self-adjoint elliptic operator of the second order. The coefficient $k$ is recovered on the base of additional integral boundary data. The existence and uniqueness of the solution to the inverse problem involving a function $u$ and a positive real number $k$ is proved.
\end{abstract}

Keywords: inverse problem, boundary value problem, second-order elliptic equations, existence and uniqueness theorem, filtration.

This paper proceeds the investigation started in $[5,6]$ and is concerned with an inverse problem for the second order differential equation

$$
-\operatorname{div}(\mathbf{k}(x, u) \nabla \psi(u))+\gamma(x, u)=f
$$

with the Dirichlet boundary data

$$
\left.u\right|_{\partial \Omega}=\beta(x)
$$

where $\mathbf{k}(x, u)$ is a matrix of functions, $\psi(u)$ and $\gamma(x, u)$ are scalar functions. We assume that $\Omega$ is a bounded domain in $\mathbb{R}^{n}$ with the boundary $\partial \Omega \subset C^{2}$.

Applications of this problem deal with the recovery of unknown parameters indicating physical properties of a medium (the heat conductivity, the permeability of a porous medium, etc.). Various aspects of such problems are discussed in [1-8] (see also the references given there). Of special interest is the problem of finding the leading coefficients of (0.1) given the additional boundary data on $\partial \Omega$ or a part of $\partial \Omega$. In $[1,2,7,8]$ this problem is investigated in the case where $\psi(u)=u, \gamma(x, u) \equiv 0, \mathbf{k}(x, u)=k(x) \mathbf{E}$, a function $k(x)$ is unknown, $\mathbf{E}$ is the identical matrix.

In this paper we study the problem of the identification of the constant coefficient $k$ in equation

$$
-k\{\mathrm{~d} i v(\mathcal{M}(x) \nabla \psi(u))+m(x) \psi(u)\}+g(x) u=f(x), \quad x \in \Omega,
$$

under the boundary data $(0.2)$. Here $\psi(u)$ is a known function, $\mathcal{M}(x)$ is a matrix of functions $m_{i j}(x), i, j=1,2, \ldots, n$. The functions $m(x), f(x), g(x), \beta(x)$ are given. In physical context the constant coefficient $k$ is interpreted as the average conductivity of a medium.

As an additional data for the recovery of the coefficient $k$ we take the condition of overdetermination

$$
k \int_{\partial \Omega} \frac{\partial \psi(u)}{\partial \bar{N}} \omega d s=\varphi
$$

where $\partial u / \partial \bar{N} \equiv(\mathcal{M}(x) \nabla u, \mathbf{n})_{R}$ is the conormal derivative, $\mathbf{n}$ is a unit outward normal to $\partial \Omega, \omega$ is a given function, $\varphi$ is a given real number. Physically, (0.4) describes, for instance, the total flux of the liquid through the surface of the rock [3]. Inverse problems with similar nonlocal boundary

*lubanova@mail.ru

(c) Siberian Federal University. All rights reserved 
conditions for elliptic equations were considered in $[5,6]$. The results of $[6]$ are concerned with the existence and uniqueness of the solution to the problem $(0.2)-(0.4)$ where $\psi(\rho)=(p-1)^{-1}|\rho|^{p-2} \rho$ and $p>2$ is a real number. The inverse problem for linear elliptic equation $(0.3)$ with $\psi(\rho)=\rho$ is discussed in [5].

The goal of our paper is to establish the existence and uniqueness of a solution to the nonlinear problem $(0.2),(0.3),(0.4)$. We discuss it in Section 2. In Section 3 we present examples and comments to the results of Section 2 and the inverse problem involved.

\section{The preliminaries}

We start with preliminary results for the direct problem $(0.2),(0.3)$. The results concern the existence, uniqueness and certain properties of the solution to this problem.

From now on we keep the notations: $\|\cdot\|_{R},(\cdot, \cdot)_{R}$ is the norm and the inner product of $\mathbb{R}^{n}$; $\|\cdot\|,(\cdot, \cdot)$ is the norm and the inner product of $L^{2}(\Omega) ;\|\cdot\|_{j},\langle\cdot, \cdot\rangle_{1}$ is the norm of $W_{2}^{j}(\Omega), j=1,2$, and the duality relation between $\stackrel{\circ}{W_{2}^{1}}(\Omega)$ and $W_{2}^{-1}(\Omega)$, respectively; $a(x)$ is the solution of the Dirichlet problem

$$
-\operatorname{div}(\mathcal{M}(x) \nabla \psi(a))+m(x) \psi(a)=0, \quad x \in \Omega,\left.\quad a\right|_{\partial \Omega}=\beta(x) ;
$$

$b(x)$ is the solution of the Dirichlet problem

$$
-\mathrm{d} i v(\mathcal{M}(x) \nabla b)+m(x) b=0, \quad x \in \Omega,\left.\quad b\right|_{\partial \Omega}=\omega(x) .
$$

We introduce the linear operator $M: W_{2}^{1}(\Omega) \rightarrow\left(W_{2}^{1}(\Omega)\right)^{*}$ of the form $M=-\operatorname{div}(\mathcal{M}(x) \nabla)+$ $m(x) I$ where $I$ is the identity operator and reckon that the following assumptions hold throughout the paper.

I. The operator $M$ is elliptic. That is, $m(x), m_{i j}(x), \partial m_{i j} / \partial x_{l}, i, j, l=1,2, \ldots, n$ are bounded, $m(x) \geqslant 0$ and there exist positive constants $m_{1}, m_{2}$ such that for any $\bar{\xi} \in \mathbb{R}^{n}$

$$
m_{1} \sum_{i=1}^{n} \xi_{i}^{2} \leqslant \sum_{i, j=1}^{n} m_{i j}(x) \xi_{i} \xi_{j} \leqslant m_{2} \sum_{i=1}^{n} \xi_{i}^{2} .
$$

II. The operator $M$ is self-adjoint. That is, $m_{i j}(x)=m_{j i}(x)$ for $i, j=1, \ldots, n$.

III. The function $\psi(\rho)$ is a continuous mapping of $(-\infty,+\infty)$ onto itself . For any $\rho_{1}, \rho_{2} \in$ $(-\infty,+\infty), \rho_{1} \neq \rho_{2}$,

$$
\left(\psi\left(\rho_{1}\right)-\psi\left(\rho_{2}\right)\right)\left(\rho_{1}-\rho_{2}\right)>0,
$$

that is, the function $\psi(\rho)$ is strictly monotone.

From the assumption III it follows that there exists an inverse $\psi^{-1}(\rho)$ of $\psi(\rho)$. The inverse $\psi^{-1}(\rho)$ is strictly monotone and continuous on $(-\infty,+\infty)$.

By the assumptions I-III the problem (1.1) has a unique solution $a$ and $\psi(a) \in W_{2}^{2}(\Omega)$ when $\psi(\beta) \in W_{2}^{3 / 2}(\partial \Omega)$. If in addition $\beta \in L^{\infty}(\partial \Omega)$, then, by the continuity of $\psi$ and Theorem 13.1 of $\left[9\right.$, Chapter 3], $\psi(a) \in L^{\infty}(\Omega)$. This means that $a \in L^{\infty}(\Omega)$.

Lemma 1.1. Let the assumptions $I-I I I$ are fulfilled. Let also $k$ be a given positive number, $f \in L^{2}(\Omega), \psi(\beta) \in W_{2}^{1 / 2}(\partial \Omega), g \in C(\bar{\Omega})$ and $g \geqslant 0$ in $\bar{\Omega}$. Then the following assertions are valid.

1. There exists a unique solution $u$ of the direct problem (0.2),(0.3) such that $\psi(u) \in W_{2}^{1}(\Omega)$. 
2. If $\psi(\beta) \in W_{2}^{3 / 2}(\partial \Omega)$ and

$$
|\psi(\rho)| \geqslant c|\rho|^{p}
$$

for any $\rho \in(-\infty,+\infty)$ where $p \geqslant 1, c>0$ are constants, then $u \in L^{2 p}(\Omega) \cap W=\{v \mid \psi(v) \in$ $\left.W_{2}^{2}(\Omega)\right\}$.

3. If $g \equiv 0$ and $\psi(\beta) \in W_{2}^{3 / 2}(\partial \Omega)$, then $\psi(u) \in W_{2}^{2}(\Omega)$. If in addition $\beta \in L^{\infty}(\partial \Omega)$, then $u \in L^{\infty}(\Omega) \cap W$.

Proof. 1. Let $\tilde{a}=\psi(a)$. From (1.1) it follows that $\tilde{a}$ solutions the equation $M \tilde{a}=0$ and satisfies the boundary condition $\left.\tilde{a}\right|_{\partial \Omega}=\psi(\beta)$. Multiplying $(0.3)$ by $\tilde{\psi}(u)=\psi(u)-\tilde{a}$ in terms of the inner product of $L^{2}(\Omega)$ and integrating by parts in the first term we obtain

$$
k \int_{\Omega}\left\{(\mathcal{M} \nabla \tilde{\psi}(u), \nabla \tilde{\psi}(u))_{R}+m(x) \tilde{\psi}^{2}(u)\right\} d x+\int_{\Omega} g(u-a) \tilde{\psi}(u) d x=(-g a+f, \tilde{\psi}(u)) .
$$

The estimation of the right side of (1.6) with the help of the Friedrichs inequality

$$
\|v\| \leqslant c_{0} \operatorname{mes}^{1 / n} \Omega\left(\int_{\Omega}\|\nabla v\|_{R}^{2} d x\right)^{1 / 2} \quad \text { for } v \in \stackrel{\circ}{W_{2}^{1}}(\Omega)
$$

leads to the relation

$$
\begin{array}{r}
k \int_{\Omega}\left\{(\mathcal{M} \nabla \tilde{\psi}(u), \nabla \tilde{\psi}(u))_{R}+m(x) \tilde{\psi}^{2}(u)\right\} d x+\int_{\Omega} g(u-a) \tilde{\psi}(u) d x \leqslant \\
\leqslant \frac{k m_{1}}{2} \int_{\Omega}\|\nabla \tilde{\psi}(u)\|_{R}^{2} d x+\frac{c_{0}^{2} \operatorname{mes}^{2 / n} \Omega}{2 k m_{1}}\|g a-f\|^{2}
\end{array}
$$

whence, by $(1.3),(1.4)$,

$$
\begin{array}{r}
\int_{\Omega}\|\nabla \tilde{\psi}(u)\|_{R}^{2} d x \leqslant \frac{1}{m_{1}} \int_{\Omega}\left\{(\mathcal{M} \nabla \tilde{\psi}(u), \nabla \tilde{\psi}(u))_{R}+m \tilde{\psi}^{2}(u)\right\} d x \leqslant \\
\leqslant \frac{c_{0}^{2} \operatorname{mes}^{2 / n} \Omega}{k^{2} m_{1}^{2}}\|g a-f\|^{2} \equiv C .
\end{array}
$$

Here the positive constant $c_{0}$ depends only on $n$. (1.7) and (1.8) implies

$$
\|\tilde{\psi}(u)\| \leqslant \frac{c_{0}^{2} \mathrm{mes}^{2 / n} \Omega}{k m_{1}}\|g a-f\|
$$

and

$$
\|\psi(u)\|_{1} \leqslant\|\tilde{a}\|_{1}+C^{1 / 2} \max \left\{1, c_{0} \operatorname{mes}^{1 / n} \Omega\right\} \equiv C_{1} .
$$

To prove the existence and uniqueness of the solution of the problem $(0.2),(0.3)$ we denote $\tilde{\psi}(u)$ by $h$. In view of the definition of $\tilde{\psi}(u)$ the function $h$ satisfies the equation

$$
\tilde{M} h \equiv M h+g \psi^{-1}(h+\tilde{a})-g a=f-g a \equiv \tilde{f}
$$

and $\left.h\right|_{\partial \Omega}=0$. One can show that the operator $\tilde{M}$ as a map from $\stackrel{\circ}{W_{2}^{1}}(\Omega)$ into $W_{2}^{-1}(\Omega)$ is demi-continuous, coercive and strongly monotone. Hence, there exists a solution $h \in W_{2}^{1}(\Omega)$ of equation (1.11) (see Theorem 2.1 [10, Chapter III]). Moreover, the solution $h$ is unique. Indeed, let $h_{1}$ and $h_{2}$ be two solutions of $(1.11)$ in $\stackrel{\circ}{W_{2}^{1}}(\Omega)$. Then by $(1.3)$ and the strong monotonicity of $\tilde{M}$ we have

$$
0=\left\langle\tilde{M} h_{1}-\tilde{M} h_{2}, h_{1}-h_{2}\right\rangle_{1} \geqslant m_{1}\left\|\nabla\left(h_{1}-h_{2}\right)\right\|^{2},
$$


which implies $h_{1}=h_{2}$.

Coming back to the original problem we conclude that the problem $(0.2),(0.3)$ has a solution $u=\psi^{-1}(h) \in W$ and this solution is unique.

2. In the hypotheses of the lemma from (1.9) it follows that $u \in L^{2 p}(\Omega)$ and

$$
\|u\|_{L^{2 p}(\Omega)} \leqslant\|\psi(u)\| \leqslant \frac{c_{0}^{2} \mathrm{mes}^{2 / n} \Omega}{k m_{1}}\|g a-f\|+\|\psi(a)\| \equiv C_{2} .
$$

Multiplying (0.3) by $M \psi(u)$ in terms of the inner product of $L^{2}(\Omega)$ we obtain the equality

$$
k\|M \psi(u)\|^{2}=-(g u, M \psi(u))+(f, M \psi(u)),
$$

from which it follows by (1.9) and the Cauchy inequality that

$$
\begin{aligned}
k\|M \psi(u)\|^{2} & \leqslant \frac{1}{k}\left[\|g\|_{C(\bar{\Omega})}^{2}\|u\|_{L^{2 p}(\Omega)}^{2} \operatorname{mes}^{(p-1) / p} \Omega+\|f\|^{2}\right]+\frac{k}{2}\|M \psi(u)\|^{2} \leqslant \\
& \leqslant \frac{1}{k}\left[\|g\|_{C(\bar{\Omega})}^{2} C_{2}^{2} \operatorname{mes}^{(p-1) / p} \Omega+\|f\|^{2}\right]+\frac{k}{2}\|M \psi(u)\|^{2}
\end{aligned}
$$

whence

$$
\|M \psi(u)\| \leqslant \frac{\sqrt{2}}{k}\left[\|g\|_{C(\bar{\Omega})}^{2} C_{2}^{2} \operatorname{mes}^{(p-1) / p} \Omega+\|f\|^{2}\right]^{1 / 2} \equiv C_{2}^{\prime} .
$$

In view of (1.8), (1.10) and Theorem 5.1 of [11, Chapter 2] the last inequality implies

$$
\|\psi(u)\|_{2} \leqslant c\left(\|M \psi(u)\|+\|\psi(u)-\psi(a)\|_{1}\right)+\|\psi(a)\|_{2} \leqslant c\left(C_{2}^{\prime}+C_{1}\right)+\|\psi(a)\|_{2}(c+1)
$$

where the constant $c$ depends on $n, m_{1}, m_{2}$, vrai $\max _{\Omega}\left|\partial m_{i j} / \partial x_{l}\right|, i, j, l=1,2, \ldots, n$ and $\operatorname{mes} \Omega$. Thus, the solution $u \in L^{2 p}(\Omega) \cap W$.

3. In the hypotheses of this part of the lemma $M \psi(u) \in W_{2}^{2}(\Omega)$ and the estimate (1.13) takes the form

$$
\|\psi(u)\|_{2} \leqslant c\left(k^{-1}\|f\|+C_{1}\right)+\|\psi(a)\|_{2}(c+1) .
$$

If in addition $\beta \in L^{\infty}(\Omega)$, then the boundedness of $u$ follows from the assumption IV and Theorem 13.1 [9, Chapter 3].

Lemma 1.2. Let the assumptions of Lemma 1.1 be fulfilled and $g a-f \geqslant 0$. Then the solution $u$ of the problem (0.2), (0.3) satisfies the inequality $u \leqslant a$ for almost all $x \in \Omega$.

Proof. Let us consider the function $w=a-u$. By (0.3) and (1.1), we have

$$
-k \operatorname{div}(\mathcal{M}(x)(\nabla \psi(a)-\nabla \psi(u)))+g(x) w=g a-f .
$$

We multiply this equation by $\psi \bar{a}-\psi u$ where

$$
\bar{a}=\left\{\begin{array}{l}
u \text { if } u \leqslant a, \\
a \text { if } u>a,
\end{array}\right.
$$

in terms of the inner product of $L^{2}(\Omega)$ and integrate by parts in the first term of the left side. This yields

$$
\begin{array}{r}
k \int_{\Omega}\left[(\mathcal{M} \nabla(\psi(\bar{a})-\psi(u)), \nabla(\psi(\bar{a})-\psi(u)))_{R}+m(\psi(\bar{a})-\psi(u))^{2}\right] d x+ \\
+\int_{\Omega}(g w-g a+f)(\psi(\bar{a})-\psi(u)) d x=0 .
\end{array}
$$

By (1.3), (1.4) and the nonnegativity of $g a-f$ it follows from (1.14) that

$$
k m_{1} \int_{\Omega}(\nabla \psi(\bar{a})-\nabla \psi(u))^{2} d x \leqslant 0
$$

which implies $\nabla(\psi(\bar{a})-\psi(u))=0$. In view of $(0.2)$ and (1.1) $\left.\bar{a}\right|_{\partial \Omega}=\left.u\right|_{\partial \Omega}$. Hence $\bar{a}-u=0$ that is $u \leqslant a$ for almost all $x \in \Omega$. 


\section{The main result}

In this section we prove the existence and uniqueness theorem for the problem $(0.2),(0.3)$, (0.4). By a solution of this problem is meant the pair involving a function $u \in W$ and a positive real number $k$ which satisfy $(0.2)-(0.4)$.

Hereafter we keep the following notation: for $v_{1}, v_{2} \in W_{2}^{1}(\Omega)$

$$
\left\langle M v_{1}, v_{2}\right\rangle_{M} \equiv \int_{\Omega}\left[\left(\mathcal{M} \nabla v_{1}, \nabla v_{2}\right)_{R}+m v_{1} v_{2}\right] d x .
$$

Theorem 2.1. Let the assumptions $I-I I I$ and the condition (1.5) be fulfilled. Let also

(i) $f \in L^{2}(\Omega), \quad \psi(\beta) \in W_{2}^{3 / 2}(\partial \Omega), \quad \omega(x) \in W_{2}^{3 / 2}(\partial \Omega), \quad g(x) \in C(\bar{\Omega}) ;$

(ii) $\omega(x)$ be nonnegative in $\partial \Omega$;

$$
\begin{gathered}
0 \leqslant g(x) \leqslant g_{1}=\text { const }<+\infty \quad x \in \Omega ; \\
\Psi \equiv\langle M \nabla \psi(a), \nabla b\rangle_{M}>0 ; \\
F(x) \equiv g a-f \geqslant 0 ; \\
\Phi \equiv \varphi-(g a-f, b)>0 .
\end{gathered}
$$

Then the problem (0.2)-(0.4) has a solution $(u(x), k)$. Moreover, $u(x) \in L^{2 p}(\Omega)$ and

$$
u(x) \leqslant a(x)
$$

for almost all $x \in \Omega$. If in addition $g \equiv 0$ or the inequalities

$$
B \equiv \Psi^{-1}\langle M \psi(a), \psi(a)\rangle_{M}^{1 / 2}\langle M b, b\rangle_{M}^{1 / 2}<2
$$

and

$$
\Phi>2 c_{0} m_{1}^{-1 / 2} \operatorname{mes}^{1 / n} \Omega\|F\|(2-B)^{-1}\langle M b, b\rangle_{M}^{1 / 2}
$$

are valid, then the solution of the problem (0.2)-(0.4) is unique.

Proof. We multiply (0.3) by $b$ in terms of the inner product in $L^{2}(\Omega)$ and integrate by parts in the first term of the resulting equality twice. In view of $(0.3)$ we obtain

$$
-\varphi+k \Psi+\int_{\Omega} g(x) u b d x=\int_{\Omega} f b d x
$$

By (2.2) and the definition of $\Phi$ in (2.4) we can rewrite (2.8) as

$$
k=(\Phi+(g(a-u), b)) \Psi^{-1} .
$$

If $g \equiv 0$, then $k=\Phi / \Psi>0$ is known function. By Lemma 2.1 the problem $(0.2)$, (0.3) with such $k$ has a unique solution $u \in L^{2 p} \cap W$.

Let now $g \neq 0$ and (2.1), (2.4) holds. We introduce the operator $A$ mapping the set $\mathbf{R}^{+}$of the positive real numbers into $\mathbf{R}$ according to the following role: for any $y \in \mathbf{R}^{+}$

$$
A(y)=\left(\Phi+\left(g\left(a-u_{y}\right), b\right)\right) \Psi^{-1}
$$

where $u_{y}$ is a solution of the direct problem $(0.2),(0.3)$ with $k=y$. According to Lemma 1.1, the problem $(0.2),(0.3)$ has a unique solution $u_{y} \in W$ and hence the value of $A(y)$ defined by (2.10) is meaningful for any $y \in \mathbf{R}^{+}$. Therefore (2.9) can be consider as the operator equation

$$
k=A(k) .
$$


Following the idea of [12, Chapter 1] one can show that the problem $(0.2)-(0.4)$ has a solution if and only if the operator equation (2.11) is solvable.

According to Lemma 1.2 in the hypotheses of the theorem $3.1 u_{y} \leqslant a$ that is (2.5) is valid. By (2.2) and (2.5),

$$
0<k_{0} \equiv \frac{\Phi}{\Psi} \leqslant A(y) \leqslant \frac{|\varphi|+|(f, b)|+g_{1}\left\|u_{y}\right\|\|b\| \mid}{\Psi}
$$

for $y \in \mathbf{R}^{+}$. The left inequality in (2.12) enables to estimate $\left\|u_{y}\right\|$. As shown in Lemma 1.1, the estimate $(1.12)$ is valid for $\psi\left(u_{y}\right)$. Therefore for every $y \geqslant k_{0}$

$$
\left\|u_{y}\right\| \leqslant c^{-1}(\operatorname{mes} \Omega)^{\frac{p-1}{2 p}}\left\|\psi\left(u_{y}\right)\right\| \leqslant c^{-1}(\operatorname{mes} \Omega)^{\frac{p-1}{2 p}}\left[\frac{c_{0}^{2} \operatorname{mes}^{2 / n} \Omega}{k_{0}^{2} m_{1}^{2}}\|g a-f\|+\|\psi(a)\|\right] \equiv C_{3} .
$$

Then for $y \geqslant k_{0}$

$$
k_{0} \leqslant A(y) \leqslant \frac{|\varphi|+|(f, b)|+g_{1} C_{3}\|b\|}{\Psi} \equiv K_{0} .
$$

This inequality implies that the operator $A$ maps $\left[k_{0}, K_{0}\right]$ into itself.

Furthermore, $A$ is a continuous operator on $\left[k_{0}, K_{0}\right]$. Indeed, let $y_{1}, y_{2} \in\left[k_{0}, K_{0}\right]$ and $u_{y 1}, u_{y 2}$ be solutions to problem $(0.2),(0.3)$ with $y_{1}$ and $y_{2}$ instead of $k$, respectively. In accordance with the definition of the operator $A$, we have

$$
A\left(y_{1}\right)-A\left(y_{2}\right)=\Psi^{-1}\left(g\left(u_{y 1}-u_{y 2}\right), b\right)
$$

On the other hand, the difference $\bar{w}=u_{y 1}-u_{y 2}$ obeys equation

$$
y_{1} M\left(\psi\left(u_{y 1}\right)-\psi\left(u_{y 2}\right)\right)+g \bar{w}=\left(y_{1}-y_{2}\right) M \psi\left(u_{y 2}\right)
$$

and the boundary condition $\left.\left(u_{y 1}-u_{y 2}\right)\right|_{\partial \Omega}=0$. Multiplying $(2.16)$ by $\psi\left(u_{y 1}\right)-\psi\left(u_{y 2}\right)$ in terms of the inner product of $L^{2}(\Omega)$ and integrating by parts in the first term of the resulting equation yields

$$
\begin{array}{r}
y_{1}\left\langle M\left(\psi\left(u_{y 1}\right)-\psi\left(u_{y 2}\right)\right), \psi\left(u_{y 1}\right)-\psi\left(u_{y 2}\right)\right\rangle_{1}+\left(g \bar{w}, \psi\left(u_{y 1}\right)-\psi\left(u_{y 2}\right)\right)= \\
=-\left(y_{1}-y_{2}\right)\left\langle M \psi\left(u_{y 2}\right), \psi\left(u_{y 1}\right)-\psi\left(u_{y 2}\right)\right\rangle_{1}
\end{array}
$$

The second term of the left side of (2.17) is nonnegative by the assumption (1.4). Multiplying (0.3) for $u_{y 2}$ by $\psi_{a}=\psi\left(u_{y 2}\right)-\psi(a)$ in terms of the inner product of $L^{2}(\Omega)$ and integrating by parts in the first term one can obtain

$$
y_{2}\left\langle M \psi\left(u_{y 2}\right), \psi\left(u_{y 2}\right)\right\rangle_{M}+\left(g\left(u_{y 2}-a\right), \psi_{a}\right)=y_{2}\left\langle M \psi\left(u_{y 2}\right), \psi(a)\right\rangle_{M}+\left(f-g a, \psi_{a}\right)
$$

from where in view of $(1.4),(1.9)$ and the Cauchy inequality it follows that

$$
\left\langle M \psi\left(u_{y 2}\right), \psi\left(u_{y 2}\right)\right\rangle_{M} \leqslant\langle M \psi(a), \psi(a)\rangle_{M}+\frac{c_{0}^{2} \mathrm{mes}^{2 / n} \Omega}{y_{2}^{2} m_{1}}\|g a-f\|^{2} .
$$

The right-hand side of (2.17) can be estimated with the help of (2.5) and (2.18).

$$
\begin{aligned}
\left|y_{1}-y_{2}\right| \mid\left\langle M \psi\left(u_{y 2}\right), \psi\left(u_{y 1}\right)-\right. & \left.\psi\left(u_{y 2}\right)\right\rangle_{1} \mid \leqslant \frac{y_{1}}{2}\left\langle M\left(\psi\left(u_{y 1}\right)-\psi\left(u_{y 2}\right)\right), \psi\left(u_{y 1}\right)-\psi\left(u_{y 2}\right)\right\rangle_{1}+ \\
+ & {\left[\langle M \psi(a), \psi(a)\rangle_{M}+\frac{c_{0}^{2} \operatorname{mes}^{2 / n} \Omega}{y_{2}^{2} m_{1}}\|g a-f\|^{2}\right] \frac{\left|y_{1}-y_{2}\right|^{2}}{2 y_{1}} }
\end{aligned}
$$


Then, by (2.5), (2.18) and (2.19), the equality (2.17) yields

$$
\begin{array}{r}
y_{1}\left\langle M\left(\psi\left(u_{y 1}\right)-\psi\left(u_{y 2}\right)\right), \psi\left(u_{y 1}\right)-\psi\left(u_{y 2}\right)\right\rangle_{1} \leqslant\left[\langle M \psi(a), \psi(a)\rangle_{M}\right. \\
\left.+\frac{c_{0}^{2} \operatorname{mes}^{2 / n} \Omega}{k_{0}^{2} m_{1}}\|g a-f\|^{2}\right] \frac{\left|y_{1}-y_{2}\right|^{2}}{y_{1}} \equiv C_{4}\left|y_{2}-y_{1}\right|^{2} .
\end{array}
$$

Next, multiplying (2.16) by $b$ in terms of the inner product of $L^{2}(\Omega)$ and integrating by parts with the use of the fact that

$$
\int_{\partial \Omega} \frac{\partial\left(y_{1} \psi\left(u_{y 1}\right)-y_{2} \psi\left(u_{y 2}\right)\right)}{\partial \bar{N}} \omega d s=0
$$

we obtain

$$
\left(g\left(u_{y_{1}}-u_{y_{2}}\right), b\right)=-y_{1}\left\langle M\left(\psi\left(u_{y 1}\right)-\psi\left(u_{y^{2}}\right)\right), b\right\rangle_{M}-\left(y_{1}-y_{2}\right)\left\langle M \psi\left(u_{y^{2}}\right), b\right\rangle_{M},
$$

whence, by (2.18) and (2.20),

$$
\begin{array}{r}
\left|\left(g\left(u_{y_{1}}-u_{y_{2}}\right), b\right)\right| \leqslant\left[y_{1}\left\langle M\left(\psi\left(u_{y 1}\right)-\psi\left(u_{y 2}\right)\right), \psi\left(u_{y 1}\right)-\psi\left(u_{y 2}\right)\right\rangle_{M}^{1 / 2}+\right. \\
\left.+\left|y_{1}-y_{2}\right|\left\langle M \psi\left(u_{y 2}\right), \psi\left(u_{y 2}\right)\right\rangle_{M}^{1 / 2}\right]\langle M b, b\rangle_{M}^{1 / 2} \leqslant C_{5}\left|y_{1}-y_{2}\right| .
\end{array}
$$

Joining (3.15) and (2.22) we are led to the inequality

$$
\left|A\left(y_{1}\right)-A\left(y_{2}\right)\right| \leqslant \frac{C_{5}}{\Psi}\left|y_{1}-y_{2}\right|
$$

proving the continuity of $A$ on $\left[k_{0}, K_{0}\right]$. By Brouwer's theorem, equation (2.11) has a solution $k \in\left[k_{0}, K_{0}\right]$. This in turn implies the existence of the solution $\{u(x), k\}$ of problem $(0.2)-(0.4)$. The solution satisfies (2.5), (2.13), (2.14). Moreover, by Lemma 1.1 the estimates (1.8)-(1.12) holds with $k_{0}$ instead of $k$ in the constants $C_{1}$ and $C_{2}$.

The only point remaining concerns the uniqueness of the solution. Let $\left(u_{1}, k_{1}\right)$ and $\left(u_{2}, k_{2}\right)$ be two solutions of problem (0.2)-(0.4). By $(0.2),\left.\left(u_{1}-u_{2}\right)\right|_{\partial \Omega}=0$. Subtracting $(0.3)$ for $\left(u_{2}, k_{2}\right)$ from $(0.3)$ for $\left(u_{1}, k_{1}\right)$, we obtain

$$
k_{1} M\left(\psi\left(u_{1}\right)-\psi\left(u_{2}\right)\right)+g \tilde{u}=-\left(k_{1}-k_{2}\right) M \psi\left(u_{2}\right) .
$$

Multiplying this difference by $\psi\left(u_{1}\right)-\psi\left(u_{2}\right)$ in terms of the inner product of $L^{2}(\Omega)$, integrating by parts and applying the arguments proving (2.20) to the resulting identity one can derive a similar inequality. Namely,

$$
\begin{aligned}
\left\langleM \left(\psi\left(u_{1}\right)\right.\right. & \left.\left.-\psi\left(u_{2}\right)\right), \psi\left(u_{1}\right)-\psi\left(u_{2}\right)\right\rangle_{1} \leqslant \\
& \leqslant\left[\langle M \psi(a), \psi(a)\rangle_{M}+\frac{c_{0}^{2} \operatorname{mes}^{2 / n} \Omega}{k_{0}^{2} m_{1}}\|g a-f\|^{2}\right] \frac{\left|k_{1}-k_{2}\right|^{2}}{k_{1}^{2}} .
\end{aligned}
$$

Furthermore, we multiply (2.23) by $b$ in terms of the inner product of $L^{2}(\Omega)$. Integrating by parts and taking into account (2.21) for $y_{1}=k_{1}$ and $y_{2}=k_{2}$ we are lead to the identity

$$
\left(g\left(u_{1}-u_{2}\right), b\right)=-k_{1}\left\langle M\left(\psi\left(u_{1}\right)-\psi\left(u_{2}\right)\right), b\right\rangle_{M}-\left(k_{1}-k_{2}\right)\left[\left\langle M\left(\psi\left(u_{2}\right)-\psi(a), b\right\rangle_{M}+\Psi\right] .\right.
$$

Subtracting $(2.9)$ for $\left(u_{2}, k_{2}\right)$ from $(2.9)$ for $\left(u_{1}, k_{1}\right)$, we obtain

$$
k_{1}-k_{2}=A k_{1}-A k_{2}=\frac{\left(g\left(u_{1}-u_{2}\right), b\right)}{\Psi} .
$$


Without loss of generality we can suppose that $k_{1} \geqslant k_{2}$. Then $\left(g\left(u_{1}-u_{2}\right), b\right) \geqslant 0$. Let us rewrite $(2.25)$ as

$$
\begin{aligned}
\left(g\left(u_{1}-u_{2}\right), b\right)+ & \Psi\left(k_{1}-k_{2}\right)= \\
& =-\left\langle k_{1} M\left(\psi\left(u_{1}\right)-\psi\left(u_{2}\right)\right)+\left(k_{1}-k_{2}\right) M\left(\psi\left(u_{2}\right)-\psi(a), b\right\rangle_{M} .\right.
\end{aligned}
$$

We can estimate the right term of (2.27) with the help of (1.8), (2.14), (2.24).

$$
\begin{aligned}
-k_{1}\left\langleM \left(\psi\left(u_{1}\right)-\right.\right. & \left.\left.\psi\left(u_{2}\right)\right), b\right\rangle_{M}-\left(k_{1}-k_{2}\right)\left\langle M\left(\psi\left(u_{2}\right)-\psi(a), b\right\rangle_{M} \leqslant\right. \\
& \leqslant k_{1}\left\langle M\left(\psi\left(u_{1}\right)-\psi\left(u_{2}\right)\right), \psi\left(u_{1}\right)-\psi\left(u_{2}\right)\right\rangle_{M}^{1 / 2}\langle M b, b\rangle_{M}^{1 / 2}+ \\
+ & \left(k_{1}-k_{2}\right)\left\langle M\left(\psi\left(u_{2}\right)-\psi(a), \psi\left(u_{2}\right)-\psi(a)\right\rangle_{M}^{1 / 2}\langle M b, b\rangle_{M}^{1 / 2} \leqslant\right. \\
& \leqslant\left[\frac{2 c_{0} \Psi \operatorname{mes}^{1 / n} \Omega}{\Phi m_{1}^{1 / 2}}\|g a-f\|+\langle M \psi(a), \psi(a)\rangle_{M}^{1 / 2}\right]\langle M b, b\rangle_{M}^{1 / 2}\left(k_{1}-k_{2}\right) .
\end{aligned}
$$

Thus, (2.26)-(2.28) lead to the inequality

$$
A k_{1}-A k_{2} \leqslant \frac{1}{2}\left[\frac{2 c_{0} \mathrm{mes}^{1 / n} \Omega}{\Phi m_{1}^{1 / 2}}\|g a-f\|+\Psi^{-1}\langle M \psi(a), \psi(a)\rangle_{M}^{1 / 2}\right]\langle M b, b\rangle_{M}^{1 / 2}\left(k_{1}-k_{2}\right)
$$

proving the contractibility of the operator $A$ by (2.6) and (2.7), from which and (2.26) it follows that $k_{1}-k_{2}=0$ and in view of (1.4), (2.24) $u_{1}-u_{2}=0$ for almost all $x \in \Omega$.

The condition $\psi(u) \geqslant c|u|^{p}$ for $p>0$ provides that $u \in L^{2 p}(\Omega)$ and $\psi(u) \in W_{2}^{2}(\Omega)$. This condition can be ignored under additional assumptions on $f, \beta$ and $\psi$.

Theorem 2.2. Let the assumptions I-III and the hypotheses (i) and (ii) of Theorem 3.1 be fulfilled. Let also $\beta \in L^{\infty}(\Omega), f \geqslant 0$ and $\beta \geqslant \rho_{0}=\min \left\{0, \rho^{*}\right\}$ for almost all $x \in \partial \Omega$ where a real number $\rho^{*}$ is such that $\psi\left(\rho^{*}\right)=0$. Then the problem (0.2), (0.3), (0.4) has a solution $(u(x), k)$. Moreover, $u(x) \in L^{\infty}(\Omega)$ and

$$
\rho_{0} \leqslant u(x) \leqslant a(x)
$$

for almost all $x \in \Omega$. If in addition $g \equiv 0$ or the inequalities (2.6) and (2.7) are valid, then the solution of the problem (0.2), (0.3), (0.4) is unique.

Proof. The proof of the theorem repeats the proof of Theorem 3.1 almost at all. We need only establish (2.29) and show that the solution of the direct problem (0.2), (0.3) belongs to $W$ for every $k>0$ because in the hypotheses of the theorem this fact is not evidenced by Lemma 2.1.

We first note that by Lemma 2.1 the direct problem $(0.2),(0.3)$ has a unique solution $u$ and $\psi(u) \in W_{2}^{1}(\Omega)$ for every $k>0$. By Lemma 2.2 the solution satisfy the right inequality of (2.29). Moreover, by the maximum principle for linear elliptic equations, $\psi(a) \geqslant \psi\left(\rho_{0}\right)$ and hence $a \geqslant \rho_{0}$ for almost all $x \in \Omega$. In order to prove the left part of (2.29) we define the function

$$
\bar{u}= \begin{cases}u & u<\rho_{0} \\ \rho_{0} & u \geqslant \rho_{0}\end{cases}
$$

and multiply $(0.3)$ by $\bar{h}=\psi(\bar{u})-\psi\left(\rho_{0}\right)$ in terms of the inner product of $L^{2}(\Omega)$. Integrating by parts in the first term of the resulting identity gives

$$
k\langle M \bar{h}, \bar{h}\rangle_{M}+\int_{\Omega}\left\{k m \psi\left(\rho_{0}\right)+g \bar{u}-f\right\} \bar{h} d x=0 .
$$


In the hypotheses of the theorem the second term is nonnegative. Therefore, by (1.3), the last equality implies $\nabla \bar{h}=0$ from which we conclude that $\bar{h}=0$ for almost all $x \in \Omega$ because $\left.\bar{h}\right|_{\partial \Omega}=0$. In view of monotonicity of $\psi$ this means that $u \geqslant \rho_{0}$ for almost all $x \in \Omega$.

As mentioned above, if $\beta \in L^{\infty}(\partial \Omega)$, then $\psi(a) \in L^{\infty}(\Omega)$ by the continuity of $\psi$ and Theorem 13.1 of $\left[9\right.$, Chapter 3]. In the hypotheses of the theorem this means that $a \in L^{\infty}(\Omega)$. Hence $u \in L^{\infty}(\Omega)$ and $\|u\|_{L^{\infty}(\Omega)} \leqslant \max \left\{\left|\rho_{0}\right|,\|a\|_{L^{\infty}(\Omega)}\right\}$ in view of (2.29). Repeating the arguments led to (1.12) one can obtain the inequality

$$
\|M \psi(u)\| \leqslant \frac{\sqrt{2}}{k}\left[\|g\|_{C(\bar{\Omega})}^{2} \max \left\{\left|\rho_{0}\right|^{2},\|a\|_{L^{\infty}(\Omega)}^{2}\right\} \operatorname{mes}^{2} \Omega+\|f\|^{2}\right]^{1 / 2} \equiv C_{2}^{\prime \prime} .
$$

By (1.8), (1.9) and Theorem 5.1 of [11, Chapter 2] the last inequality implies

$$
\|\psi(u)\|_{2} \leqslant c\left(C_{2}^{\prime \prime}+C_{1}\right)+\|\psi(a)\|_{2}(c+1),
$$

which proves that the solution $u$ belongs to $W$.

Certain inverse problems can be reduced to the problem $(0.2)-(0.4)$. This allows to establish the existence and uniqueness theorems for such problems with the help of Theorems 2.1 and 2.2. In particular, the problem of the identification of the constant coefficient $\nu$ in equation

$$
-\nu \mathrm{d} i v\left(\mathcal{M}(x) \nabla \psi_{1}(v)\right)+g(x) \psi_{2}(v)=f(x)
$$

with the boundary condition $\left.v\right|_{\partial \Omega}=\mu(x)$ and the condition of overdetermination

$$
\nu \int_{\partial \Omega} \frac{\partial \psi_{1}(v)}{\partial \bar{N}} \omega d s=\varphi_{1}
$$

are reduced to the problem $(0.2)-(0.4)$ if the function $\psi_{2}(\rho)$ is an injection in $(-\infty,+\infty)$. Indeed, we can define a function $u=\psi_{2}(v)$ and rewrite this problem as $(0.2)-(0.4)$ with $k=\nu, \psi(\rho)=$ $\psi_{1}\left(\psi_{2}^{-1}(\rho)\right), \beta=\psi_{2}(\mu)$ and $\varphi=\varphi_{1}$.

The inverse problem $(0.2),(0.3)$ with the condition of overdetermination given only on a part $\Gamma$ of the boundary $\partial \Omega$, that is

$$
k \int_{\Gamma} \frac{\partial \psi(u)}{\partial \bar{N}} \omega d s=\varphi,
$$

can also be reduced to the problem $(0.2),(0.3),(0.4)$. If the function $\omega \in W_{2}^{3 / 2}(\Gamma)$ is finite on $\Gamma$ and supp $\omega \subset \Gamma$ then one can extend $\omega$ into $\partial \Omega$ setting $\omega=0$ in $\partial \Omega \backslash \Gamma$ and consider the integral in (2.31) over the whole boundary $\partial \Omega$. In this case Theorem 2.1 is formulated as follows.

Theorem 2.3. Let the assumptions $I-I I I$ and (1.5) be fulfilled, $f \in L^{2}(\Omega), \psi(\beta) \in W_{2}^{3 / 2}(\partial \Omega)$, $\omega(x) \in W_{2}^{3 / 2}(\Gamma), g(x) \in C(\bar{\Omega})$. Let also $\omega(x)$ be nonnegative and finite on $\Gamma$, supp $\omega \subset \Gamma$, $\omega(x)=0$ on $\partial \Omega \backslash \Gamma$ and (2.1)-(2.4) hold. Then the problem (0.2), (0.3), (2.31) has a solution $(u(x), k)$. Moreover, $u(x)$ satisfies (2.5) for almost all $x \in \Omega$. If in addition $g \equiv 0$ or (2.6), (2.7) are fulfilled, then the solution of the problem (0.2), (0.3), (2.31) is unique.

Theorem 2.2 can be reformulate for the problem $(0.2),(0.3),(2.31)$ in a similar manner.

Theorem 2.4. Let the assumptions I-III be fulfilled, $f \in L^{2}(\Omega), \psi(\beta) \in W_{2}^{3 / 2}(\partial \Omega), \omega(x) \in$ $W_{2}^{3 / 2}(\Gamma), g(x) \in C(\bar{\Omega}), \beta \in L^{\infty}(\Omega)$. Let also (2.1)-(2.4) hold, $f \geqslant 0$ for almost all $x \in \Omega$, the function $\omega(x)$ be nonnegative and finite on $\Gamma, \operatorname{supp} \omega \subset \Gamma, \omega(x)=0$ on $\partial \Omega \backslash \Gamma$ and $\beta \geqslant \rho_{0}$ for almost all $x \in \partial \Omega$. Then the problem (0.2), (0.3), (2.31) has a solution $(u(x), k)$. Moreover, $u(x) \in L^{\infty}(\Omega)$ and (2.29) is fulfilled for almost all $x \in \Omega$. If in addition $g \equiv 0$ or the inequalities (2.6) and (2.7) are valid, then the solution of the problem (0.2), (0.3), (2.31) is unique. 


\section{Examples and comments}

As mentioned above the interest to the identification of coefficients in the elliptic equations, among them (0.3), is due to its extensive applications. Certain examples of such problems were considered in [6]. In particular, the special case of (0.3)

$$
-k \operatorname{div}\left(\mathcal{M}(x)|u|^{p-2} \nabla u\right)=0
$$

describes the steady-state isothermal gas filtration [13] where $p=3, \mathcal{M}(x)=\left(m \mu_{1}\right)^{-1} \mathbf{E}, u$ is the pressure of a liquid in the pores, $\mu_{1}$ is the viscosity of liquid, $k$ and $m$ are the average permeability and the porosity of the rock. In this case $\psi(\rho)=(p-1)^{-1}|\rho|^{p-2} \rho$ and $g \equiv 0$. The equation (4.1) satisfies the assumptions I-III. If the other hypotheses of Theorem 2.1 coincide, then the inverse problem (3.1), (0.2), (0.4) has a unique solution.

In [14] the physical model of the non-equilibrium effects in a simultaneous flow of immiscible fluids in porous media is presented. For the steady-state flow with capillary counter-current imbibition the basic equation for the effective water saturation assumes the form

$$
k \Delta \psi(u)=0
$$

where $u$ is the actual water saturation, the coefficient $k$ depends on the air permeability of the porous medium, its porosity (the relative volume occupied by the pores), the surface tension and a (conventional) contact angle at the triple water-oil-solid boundary,

$$
\psi(\rho)=-\int_{0}^{\rho} \frac{f_{1}(s) f_{2}(s)}{f_{1}(s)+f_{2}(s)} J^{\prime}(s) d s .
$$

The dimensionless nonnegative quantities $f_{1}$ and $f_{2}$ are called the relative permeabilities and satisfy the inequality $0 \leqslant f_{i} \leqslant 1$. The function Leverett $J$ is the dimensionless capillary pressure. $f_{1}(s)$ is a monotonic non-decreasing smooth function. The function $f_{2}(s)$ is a monotonic nonincreasing smooth one. Finally, the nonnegative function $J(u)$ monotonically decreases. The function $\psi$ is continuous and strictly increases on $(-\infty,+\infty)$. Therefore under the hypotheses of Theorem 3.2 the inverse problem $(0.2)$, (0.4), (3.2) has a unique solution $(u(x), k)$.

Some kinds of equations (2.30) are involved in modeling the electric fields of semiconductors. In the absence of an external electric field the stationary nonlinear dissipative equation for the potential $u$ of the self-consistent electric field of a semiconductor takes the form [15]

$$
-k \Delta\left(u+\frac{\varepsilon}{2} u^{2}+\frac{\varepsilon^{2}}{6} u^{3}\right)+\lambda|u|^{q} u=0
$$

where the positive parameters $k$ and $\lambda$ depends on the electric susceptibility of the semiconductor, the parameter $\varepsilon>0$ depends on the temperature of free electrons, $q \geqslant 0$. These equations can be reduced to $(0.3)$ with the appropriate function $\psi(\rho)$ satisfying the assumption IV.

The last point of our considerations is the stability of the solution to $(0.2),(0.3),(0.4)$ with respect to $\varphi$. One can prove it much as the contractibility of the operator $A$ was established in Theorem 3.1.

\section{References}

[1] G.Alessandrini, R.Caburro, The Local Calderon Problem and the Determination at the Boundary of the Conductivity, Comm. Partial Differential Equations, 34(2009), 918-936.

[2] A.P.Calderon, On an inverse boundary value problem, Seminar on Numerical Analysis and its Applications to Continuum Physics, Rio de Janeiro, Brazil, 1980, Soc. Brazil. Mat., Rio de Janeiro, 1980, 65-73. 
[3] A.Hasanov, A.Erdem, Determination of unknown coefficient in a nonlinear elliptic problem related to the elasto-plastic torsion of a bar, IMA J. Appl. Math., 73(2008), no. 4, 579-591.

[4] M.V.Klibanov, A.Timonov, Carleman Estimates for Coefficient Inverse Problems and Numerical Applications, VSP, Utrecht, Netherlands, 2004.

[5] A.Sh.Lyubanova, Identification of a constant coefficient in an elliptic equation, Appl. Anal., 87(2008), 1121-1128.

[6] A.Sh.Lyubanova Identification of a constant coefficient in a quasi-linear elliptic equation Journal of inverse and ill-posed problems, 22(2014), 341-356.

[7] A.Nachman, B.Street, Reconstruction in the Calderon Problem with Partial Data, Communications in Partial Differential Equations, 35(2010), 37-390.

[8] G.Nakamura, K.Tanuma, A nonuniqueness Theorem for Inverse boundary Value Problem in Elasticity, SIAM J. Appl. Math., 56(1996), 602-610.

[9] O.A. Ladyzenskaja, N.N. Uralceva, Linear and Quasilinear Equations of Elliptic Type, Academic Press, New York, 1973.

[10] H.Gajewski, K.Gröger, K. Zacharias, Nichtlinear Operatorgleichungen und Operatordifferentialgleichungen, Mathematische Lehrbücher und Monographien, II, Abteilung, Mathematische Monographien, vol. 38, Akademie-Verlag, Berlin, 1974.

[11] J.-L.Lions, E.Magenes, Problemes aux Limites Non Homogenes et Applications, vol. 1, Travaux et Recherches Mathematiques, no. 17, Dunod, Paris, 1968.

[12] A.I.Prilepko, D.G.Orlovsky, I.A.Vasin, Methods for solving inverse problems in mathematical physics, Marcel Dekker Inc., New York, 2000.

[13] G.I. Barenblatt, V.M.Entov, V.M.Ryzhik, Flow of fluids through natural rocks, Kluwer Academic Publ., 1990.

[14] G.I.Barenblatt, J.Garcia-Azorero, A.De Pablo, J.L.Vazquez, Mathematical Model of the Non-Equilibrium Water-Oil Displacement in Porous Strata, Applicable Analysis, 65(1997), $19-45$.

[15] A.G.Sveshnikov, A.B.Alshin, M.O.Korpusov, Yu.D.Pletner, Linear and nonlinear equations of the Sobolev type, Physmatlit, Moskow, 2007 (in Russian).

\section{Об одной обратной задаче для квазилинейного эллиптического уравнения}

Анна Ш. Любанова

$\overline{И с с л е д у е т с я ~ з а д а ч а ~ и д е н т и ф и к а ц и и ~ н е и з в е с т н о г о ~ п о с т о я н н о г о ~ к о э ф ф и ц и е н т а ~ в ~ с т а р ш е м ~ ч л е н е ~}$ уравнения с частными производными $-k M \psi(u)+g(x) u=f(x)$ при граничном условии Дирихле. Здесъ $\psi(u)$ - нелинейная возрастающая функиия от $u, M$ - линейный самосопряженный эллиптический оператор второго порядка. Коэффициент $k$ восстанавливается по дополнительным интегральным данным на гранище. Доказывается существование и единственность решения обратной задачи, включающего функиию и и положительное действительное число $k$.

Ключевые слова: обратная задача, краевая задача, эллиптическое уравнение второго порядка, теорема существования и единственности, фильтрация. 